\title{
Soft Nucleation of an Elastic Crease
}

\author{
P. Ciarletta ${ }^{1}$ and L. Truskinovsky ${ }^{2}$ \\ ${ }^{1}$ MOX Laboratory, Dipartimento di Matematica, Politecnico di Milano, 20133 Milano, Italy \\ ${ }^{2}$ ESPCI ParisTech, PMMH, CNRS-UMR 7636, 75005 Paris, France
}

(Received 21 February 2019; revised manuscript received 26 April 2019; published 18 June 2019)

\begin{abstract}
Creasing instability is ubiquitous in soft solids; however, its inception remains enigmatic as it cannot be captured by the standard linearization techniques. It also does not fit the conventional picture of a barriercrossing nucleation, and instead carries some features of a second order phase transition. Here we show that despite its fundamentally nonlinear nature, creasing has its origin in marginal stability which is, however, obscured by the dominance of long-range elastic interactions. We argue that despite its supercritical (soft) character, creasing bifurcation can be identified by the condition that the (generalized) driving force acting on an incipient stress singularity degenerates. The analytic instability criterion, obtained in this way, shows an excellent agreement with both physical experiments and direct numerical simulations.
\end{abstract}

DOI: 10.1103/PhysRevLett.122.248001

Creasing is a highly localized mechanical instability in soft elastic solids (gels, elastomers, tissues). It manifests itself through the appearance, on a free surface of a precompressed body, of a singular region of cusped folding and culminates in the formation of a zone of self-contact [1-5]. Creasing is encountered in a wide range of applications from biomedical coating [6] and constrained swelling [7,8] to tunable adhesion [9]. It is of significant theoretical interest as an example of nonlinearizable instability [10-14] which shows universality: the creasing strain was found to be insensitive to the constitutive behavior [15], the geometry of the body [16], and the type of loading $[17,18]$.

Creasing instability is similar to buckling [19] and wrinkling [20] because it relies mainly on geometrical nonlinearities of an elastic solid [21]. However, in the case of creasing, while the associated displacements are small, the displacement gradients are large which disqualifies standard perturbation methods $[22,23]$ and suggests that stability is lost in the strong sense [24]. This type of instability is characteristic for systems where the order parameter is a gradient, which implies nonlocality and dominance of long-range interactions [25-27].

In the presence of an internal length scale, creasing displays features of nucleation and growth phenomena usually associated with first order phase transitions [21,28] and microcracking [29]. However, in the scale-free setting of classical elasticity, creasing instability lacks traditional "domain boundary" structure and resembles a second order phase transition [30]. This implies "soft" nucleation which is closer to a barrierless binodal transition than to a spinodal transition. Despite the progress achieved in the numerical studies of creasing instability in a regularized setting $[10,21,22,31]$, the physical understanding of its purely elastic, scale-free nature remains limited.
In this Letter we pursue a simple idea that the creation of an incipient, highly localized self-contact zone requires an energy inflow from far away where the necessary work is done by the applied forces. To quantify this picture we introduce an energetic criterion of creasing which follows a closely similar approach in fracture mechanics $[32,33]$. An (incomplete) analogy between the two subjects is suggested by the observation that a crack is a singular set of the deformation gradient while a crease is a singular set of the inverse deformation gradient.

We argue that despite its supercritical (soft) character, creasing bifurcation can be identified by the condition that the (generalized) driving force acting on an incipient stress singularity becomes degenerate. To compute such driving force we use the elastic energy-momentum tensor [34]. It allows us to link the energy released globally as a result of the formation of a crease with the energy required locally to bring together the two sides of a zone of self-contact.

By associating the creasing threshold with the condition that the energy fluxes at macro and micro scale are exactly balanced, we reproduce in the creasing framework the original idea of Griffith [35,36]. Quite remarkably, our analytic Griffith-type instability condition, obtained without knowing the exact solution of the nonlinear elasticity problem, shows an excellent agreement with both physical experiments and direct numerical simulations.

Denote by $x_{i}$ and $X_{a}$ (with $i, a=1,2,3$ ) the Cartesian coordinates of the points of an elastic body in the current and reference configurations. We assume that $x_{3} \equiv X_{3}$ and focus on the behavior of a $2 \mathrm{D}$ body $\Omega=\{[-L, L] \times[0, L]\}$. Suppose that the material is incompressible, so that $\operatorname{det} \mathbf{F}=1$, where $\mathbf{F}_{i a}=\partial \mathbf{x}_{i} / \partial \mathbf{X}_{a}$ and now $i, a=1,2$. Denoted by $\mathcal{W}=\int_{\Omega} w d^{2} X$ the elastic energy of the body, where $w=w(\mathbf{F})$ is the energy density. Under the assumption that the material is isotropic, the function $w$ 
can depend only on $I=\operatorname{tr}\left(\mathrm{F}^{T} \mathrm{~F}\right)$ [37] and we assume, for simplicity, that this dependence is convex. Consider next the simplest problem where the body $\Omega$ is uniformly compressed in a horizontal direction in a hard device. More precisely, we assume that $x_{1}=\lambda X_{1}$ at $\left|X_{1}\right|=L$, where $\lambda \leq 1$ is the applied stretch. We also assume that $x_{2}$ remains unconstrained at $\left|X_{1}\right|=L$ in the sense that $P_{12}=0$ where $P_{a j}$ are the components of the Piola-Kirchhoff stress tensor

$$
\mathbf{P}=\partial w / \partial \mathbf{F}-p \mathbf{F}^{-1}
$$

and $p$ (kinematic pressure) is the Lagrange multiplier enforcing the incompressibility constraint. At the free surface $X_{2}=0$ we impose the zero tractions condition $\mathbf{P}^{T} \mathbf{e}_{2}=0$ where $\mathbf{e}_{2}=(0,1)$. We also assume that the vertical displacements at the surface $X_{2}=L$ are constrained so that $x_{2}=L / \lambda$ while $P_{21}=0$. Our task is to find the critical value $\lambda=\lambda_{c}<1$ when the homogeneous configuration $x_{1}=\lambda X_{1}, x_{2}=X_{2} / \lambda$ becomes unstable vis a vis the formation of an isolated crease with infinitesimal length $\delta \ell \ll L$, see Fig. 1 .

(a)

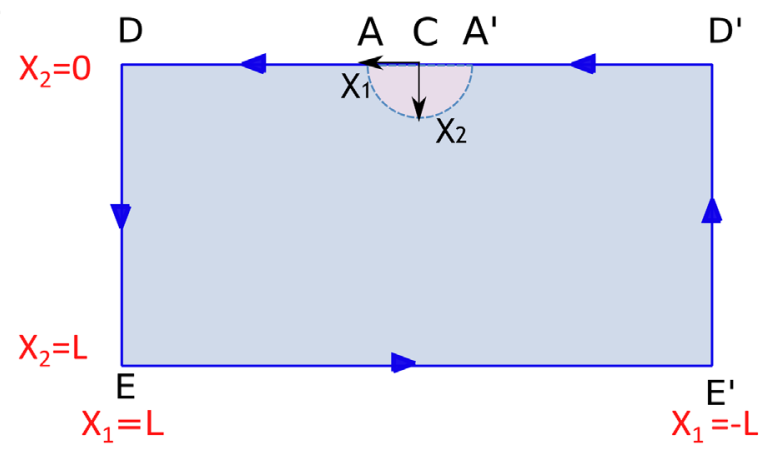

(b)

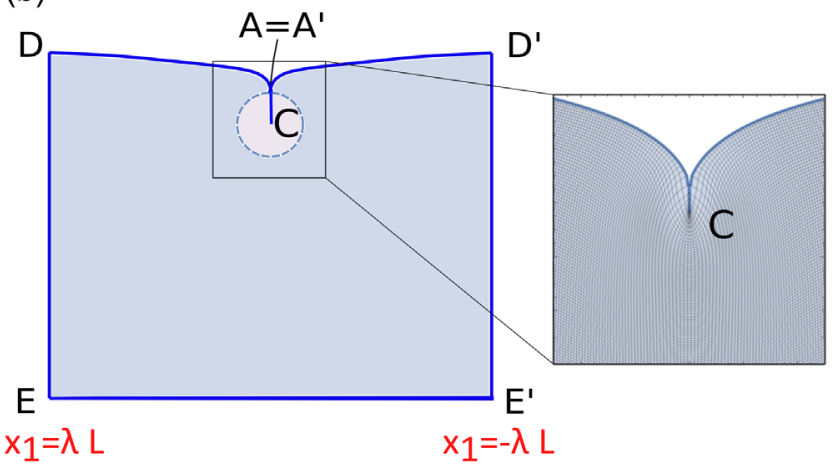

FIG. 1. Sketch of the (a) reference and (b) actual configurations of a creased elastic slab subjected to a compressive stretch $\lambda$ in the horizontal direction. The blue line indicates the (material) path $\gamma$ used to compute the energy flux. Shaded areas show the internal solution. An inset in (b) illustrates the critical deformed shape (intermediate asymptotics) obtained analitically for the neoHookean material with a crease with a self-contact length $\delta \ell \ll L$.
The equilibrium problem reduces to solving the system of equations $\nabla \cdot \mathrm{P}=0$; however, even without knowing the creasing solution of this nonlinear problem, one can find its internal (near field) $|\mathbf{X}| \sim \delta \ell$, external (far field) $|\mathbf{X}| \sim L$ and intermediate $\delta \ell \ll|\mathbf{X}| \ll L$ asymptotics $[30,38]$.

The far field asymptotics is not affected by the appearance of the crease, and therefore the external solution represents the original homogeneous deformation. It is fully characterized by the horizontal uniaxial stress $P_{11}(\lambda)=2 w^{\prime}\left(I_{\lambda}\right)\left(\lambda-\lambda^{-3}\right)$ and the pressure $p=2 w^{\prime}\left(I_{\lambda}\right) \lambda^{2}$, where $I_{\lambda}=\left(\lambda^{2}+\lambda^{-2}\right)$; other stress components disappear, $P_{12}=P_{21}=P_{22}=0$.

The near field asymptotics is furnished by the canonical mapping from a half space to the whole space (with the formation of a line of self-contact). Using the conditions of incompressibility and equilibrium we obtain $[21,39,40]$ : $x_{1}=R / \sqrt{2} \sin (2 \Theta), \quad x_{2}=R / \sqrt{2} \cos (2 \Theta)$, where $R=$ $\sqrt{\left(X_{1}\right)^{2}+\left(X_{2}\right)^{2}}$ and $\Theta=\tan ^{-1}\left(X_{1} / X_{2}\right)$. The pressure distribution, $p_{\text {in }}(R)=w^{\prime}(5 / 2)(1-3 \log R)-C$, is defined up to an additive constant $C$ which controls how much the two sides of the self-contact line with a small length $\delta \ell$ in the reference configuration are pushed against each other. To match at the order $\epsilon=\delta \ell / L$ the average stress exchanged along the vertical line bisecting the crease with the homogeneous horizontal stress in the far field $P_{11}(\lambda)$, we must choose $C=\lambda P_{11}(\lambda)-3 w^{\prime}(5 / 2) \log \delta \ell$ [41].

The opposite sides of the self-contact must be pulled together by forces distributed on the reference surface with the normal $-\mathbf{e}_{2}$. This force distribution in the "internal" problem must be effectively canceled by the matching distribution of pushing forces in the "intermediate" problem. Consider a domain with characteristic length $\bar{L}$ centered around the incipient crease, such that $\delta \ell \ll \bar{L} \ll L$. In the limit $\epsilon \rightarrow 0$ the elastic field in this domain will be affected by the crease only through the resultant of such pushing force distribution $\delta f=-4 w^{\prime}\left(I_{\lambda}\right)\left(\lambda^{2}-\lambda^{-2}\right) \delta \ell$.

Since this forcing is weak, we can obtain the intermediate asymptotics by linearizing the elastic problem around the homogeneous external solution. To this end we assume that $\mathbf{x}=\mathrm{F}_{o} \mathbf{X}+\delta \mathbf{u}$, with $\mathrm{F}_{o}=\operatorname{diag}(\lambda, 1 / \lambda)$. The displacement field $\delta \mathbf{u}$ is incompressible and can be written as $\delta u_{1}=-\delta \phi_{, 2} \lambda^{-1} \delta u_{2}=\delta \phi_{, 1} \lambda$, where commas denote partial derivatives. The stream function $\delta \phi(\mathbf{x})$ satisfies a biharmonic equation with coordinates rescaled by the factor [41]

$$
s=\sqrt[4]{\frac{\alpha+\lambda^{4}+1-\sqrt{\alpha^{2}+2 \alpha\left(\lambda^{4}+1\right)+\left(\lambda^{4}-1\right)^{2}}}{2}}
$$

where

$$
\alpha=2\left(w^{\prime \prime}\left(I_{\lambda}\right) / w^{\prime}\left(I_{\lambda}\right)\right)\left(\lambda^{3}-\lambda^{-1}\right)^{2} .
$$


In the case $\lambda=1$, the Green's function $G(\mathbf{x})$ for this problem is classical [42] and its generalization for the case $\lambda<1$ is straightforward; here we only mention that it depends on physical nonlinearity weakly, through a scaling coefficient $w^{\prime \prime}\left(I_{\lambda}\right) / w^{\prime}\left(I_{\lambda}\right)[41]$.

We can then write $\delta \phi=G \delta f$. To obtain the first corrections to this asymptotic result at $\epsilon$ that is small but finite, it is sufficient to uniformly distribute the force $\delta f$ over the interval of the length $2 \delta \ell: \delta \phi_{1}(\mathbf{X})=$ $[\delta f /(2 \delta \ell)] \int_{-\delta \ell}^{\delta \ell} G\left(X_{1}-\zeta, X_{2}\right) d \zeta$. An explicit expression of the displacement field in this approximation can be found in the Supplemental Material [41].

The next step is to compute the energy increment $\Delta \mathcal{W}$ associated with the infinitesimal advance of the tip of a crease $\delta \ell$. We can write: $\Delta \mathcal{W}=J(\delta \ell) \delta \ell$, where the driving force is $J(\delta \ell)=\mathbf{J}(\delta \ell) \mathbf{m}$ and $\mathbf{m}$ is the direction of the advance of the crease. To compute the energy flux we can use the well known formula $\mathbf{J}(\delta \ell)=\int_{\gamma} \mathrm{T}(\delta \ell) \mathbf{n} d s$ [43-45], where

$$
\mathbf{T}=w \mathbf{l}-(\partial w / \partial \mathbf{F}) \mathbf{F}
$$

is the energy-momentum (Eshelby) tensor [46] and I is the unit tensor. In the definition of the driving force, the element of length $d s$ is taken along the contour $\gamma$ following the body boundary $\partial \Omega$ and surrounding the crease with the normal $\mathbf{n}$. We recall that due to Noether identity [47] $\nabla \cdot T=0$ and therefore the integral in the definition of $\mathbf{J}$ is path independent. In our case $\mathbf{m}=(0,1)$ and $J=\int_{\gamma}\left(w n_{2}-P_{i j} n_{i} \mathrm{~F}_{j 2}\right) d s$. The anticlockwise path $\gamma$ is depicted by the blue line in Fig. 1(a).

We start from the external problem which does not see the crease. The contributions to the $J$ integral along the vertical segments $D E, D^{\prime} E^{\prime}$ are equal to zero, because in the external asymptotics $P_{12}=0$ and $x_{1,2}=0$ at $\left|X_{1}\right|=L$. The contribution along $E E^{\prime}$ takes the form: $\left.J\right|_{E E^{\prime}}(0)=$ $-\int_{-L}^{L} w\left(I_{\lambda}\right) d X_{1}=-2 L w\left(I_{\lambda}\right)$, where we used the fact that $P_{21}=P_{22}=0$ at $X_{2}=L$. Since, in the absence of singularities, the $J$ integral around a closed contour must vanish, we conclude that $\left.J\right|_{D^{\prime} D}(0)=-\left.J\right|_{E E^{\prime}}(0)=2 L w\left(I_{\lambda}\right)$.

The compatibility of external and internal problems requires that $\left.J\right|_{D^{\prime} D}(0)=\left.J\right|_{D^{\prime} D}(\delta \ell)$, where the term $\left.J\right|_{D^{\prime} D}(\delta \ell)$ can be split into contributions from the parts of the reference surface with and without self-contact. Therefore $\left.J\right|_{D^{\prime} D}(\delta \ell)=\left.J\right|_{A^{\prime} A}(\delta \ell)+\left.2 J\right|_{A D}(\delta \ell)$ where we used the fact that $\left.J\right|_{A D}(\delta \ell)=\left.J\right|_{D^{\prime} A^{\prime}}(\delta \ell)$ by symmetry. We may then define $\Delta \mathcal{W}^{+}=\left[\left.J\right|_{A^{\prime} A}(\delta \ell)-\left.J\right|_{A^{\prime} A}(0)\right] \delta \ell \geq$ 0 as the incremental energy "absorbed" by the self-contact, and $\Delta \mathcal{W}^{-}=\left(\left.2 J\right|_{A D}(\delta \ell)-\left.2 J\right|_{A D}(0)\right) \delta \ell \leq 0$ as the incremental energy "released" due to unloading in the rest of the body. In the absence of an analog of the conventional toughness, the critical condition of the crease initiation takes the form

$$
\lim _{\delta \ell \rightarrow 0} \frac{\Delta \mathcal{W}^{+}+\Delta \mathcal{W}^{-}}{(\delta \ell)^{2}}=0
$$

To compute the "absorbed" energy $\Delta \mathcal{W}^{+}$we need to evaluate the $J$ integral for the self-contacting portion of the free surface (using the internal solution). We obtain $\Delta \mathcal{W}^{+}=$ $\delta \ell \int_{-\delta \ell}^{\delta \ell}\left[w(5 / 2)-P_{21} F_{12}\right] d X_{1}$. Then $\lim _{\delta \ell \rightarrow 0} \Delta \mathcal{W}^{+} /(\delta \ell)^{2}=$ $2\left[w(5 / 2)-2 w^{\prime}\left(I_{\lambda}\right)\left(\lambda^{2}-\lambda^{-2}\right)\right]$, and since $w$ is non-negative and is also a convex function of $I$, this expression is positive for all $\lambda<1$. Note also that, despite the presence of a singularity at the tip of the crease, it is too weak (logarithmic) to generate a special contribution to the $J$ integral.

To compute the "released" energy $\Delta \mathcal{W}^{-}$, we can use the intermediate asymptotics. The goal is to evaluate the integral $\left.J\right|_{A D}$. However, we begin with computing the integral $\left.J\right|_{B D}$ where $B$ is a point on the top surface of the body with coordinates $(\bar{L}, 0)$. We recall that $\bar{\epsilon}=\bar{L} / L \ll 1$ and $\epsilon \ll \bar{\epsilon}$.

Given that the normal tractions vanish along the path $B D$, we obtain for the incremental stress $\delta p_{12}=\delta p_{22}=0$, hence $\left.J\right|_{B D}=\left.\int_{\bar{L}}^{L} w\right|_{X_{2}=0} d X_{1}$. We can now expand the energy $w$ up to the first order in $\epsilon$ : $\left.\int_{\bar{L}}^{L} w\right|_{X_{2}=0} d X_{1}=$ $w\left(I_{\lambda}\right)(L-\bar{L})-2 P_{11}(\lambda) \delta u_{1}(\bar{L}, 0)+o(\epsilon)$ where we used the incompressibility constraint and the fact that in the external asymptotics $\delta u_{1}(L, 0)=0$. Note that $w\left(I_{\lambda}\right)(L-\bar{L})=w\left(I_{\lambda}\right) L(1-\bar{\epsilon}) \sim w\left(I_{\lambda}\right) L$. Since $\delta u_{1}\left(X_{1}, 0\right)$ is constant for $X_{1} \geq \delta \ell$ [41], we can replace $\delta u_{1}(\bar{L}, 0)$ by $\delta u_{1}(\delta \ell, 0)$. Therefore the computed asymptotics for $\left.J\right|_{B D}$ can be also used to evaluate $\left.J\right|_{A D}$ and the "released" energy can be written as $\Delta \mathcal{W}^{-}=-2 P_{11}(\lambda) \delta u_{1}(\delta \ell, 0) \delta \ell$. This expression is always negative since both $P_{11}$ and $\delta u_{1}$ are negative for $\lambda<1$. Finally, combining all the obtained formulas [41] we obtain $\lambda_{c}$ as a real root of the equation

$$
\begin{aligned}
& -\left(\frac{w(5 / 2)}{2 w^{\prime}\left(I_{\lambda}\right)}-\left(\lambda^{2}-\lambda^{-2}\right)\right) \\
& +\frac{\left(\lambda-\lambda^{-3}\right)\left(-\lambda^{4}+2 s^{2}-1\right) \lambda s^{3}\left(\lambda^{4}-1\right)\left(\lambda^{4}+s^{4}\right)}{\lambda s\left(s^{4}-\lambda^{2}\right)\left\{\lambda^{4}+s^{8}+s^{4}\left[\lambda^{6}+\left(\lambda^{2}-1\right) \alpha+3 \lambda^{2}-2\right]\right\}}=0,
\end{aligned}
$$

where $s$ is given in Eq. (1).

Note that in contrast to the case of fracture where the analog of our $\Delta \mathcal{W}^{-}$is linear in $\delta \ell$ and the stability condition concerns the first variation of the energy, in the case of creasing, $\Delta \mathcal{W}^{-}$is of the same order $(\delta \ell)^{2}$ as the bulk energy increment $\Delta \mathcal{W}^{+}$. Therefore the equilibrium condition is effectively formulated in terms of the second variation; the latter should be then understood in the strong sense [27]. The absence of scale makes the implied generalized bifurcation (from a homogeneous state) supercritical and global, while it is subcritical and local in the case of microcracking. Creasing is also different from the scale-free homogenous nucleation of dislocations, which is 


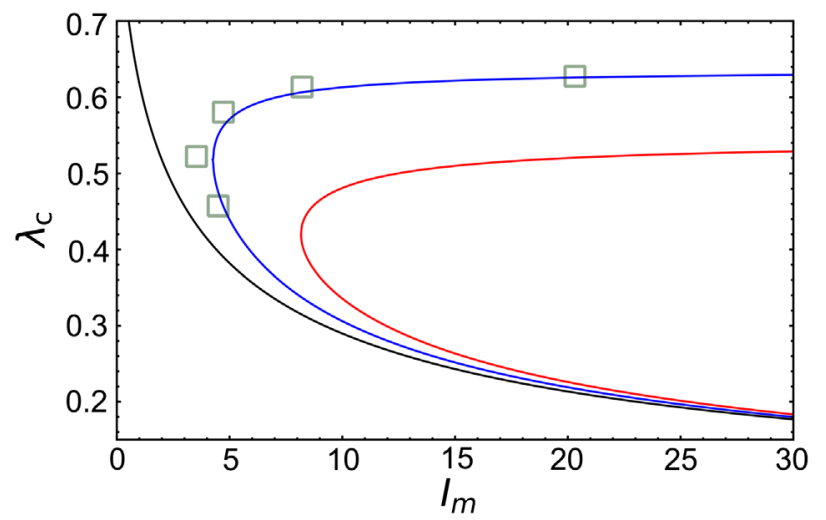

FIG. 2. Stability limits: creasing threshold (this Letter)—blue; Biot threshold-red, inextensible limit-black. Squares: FEM simulations [49].

usually associated with linear instability of spinodal type [48].

To illustrate our main result [Eq. (3)] and to clarify the role of physical nonlinearity $[49,50]$ we have chosen the Gent's energy density [51]

$$
w(I)=-\frac{\mu I_{m}}{2} \log \left(1-\frac{I-2}{I_{m}}\right)
$$

where $\mu$ is a characteristic stress, and $I_{m}>1$ is a maximum deformability, defined for instance by the average contour length of the fiber chains composing a polymeric network. Note that Gent's model is strongly elliptic as long as $\mu I_{m}>0$, so $\mu$ should be of the same sign as $I_{m}$. Gent's model reduces to the classical neo-Hookean model for elastomers when $I_{m} \rightarrow \infty$; this limit is degenerate since then $w^{\prime \prime}\left(I_{\lambda}\right) / w^{\prime}\left(I_{\lambda}\right)=\left[I_{m}-\left(I_{\lambda}-2\right)\right]^{-1} \rightarrow 0$ and only geometric nonlinearity remains in the problem [as far as the roots of Eq. (3) are concerned].

The dependence of the root $\lambda_{c}$ on $I_{m}$ is illustrated in Fig. 2 (blue line). Observe an interesting reentry behavior of stability boundary that is caused by rapidly decreasing extensibility of the material at high compressive stretches (the effect of physical nonlinearity). The obtained results are in a very good agreement with finite element simulations reported in Ref. [49], which concerns both the value of $\lambda_{c}$ and the fact that creasing does not occur below $I_{m} \sim$ 3.7 (cf. with the value $I_{m} \sim 3.1$ in Ref. [49]). Our results also agree with experimental measurements, which report critical values of $\lambda$ at about 0.63 for rubber [1], 0.635 for silicone [5], and 0.65 for polymer gels [15]. Note also that for neo-Hookean material, the threshold $\lambda_{c} \simeq 0.6362$ obtained in this Letter is close to the upper bound $\lambda_{c} \simeq$ 0.6372 recently computed in Ref. [38].

A similar reentry behavior is also exhibited by the Biot threshold [20] which identifies conditions of linear instability of a prestressed solid body with a free surface. Being a strong (nonlinear) instability, creasing always precedes weak Biot instability independently on whether we approach it by loading or unloading. For Gent's material, Biot threshold can be obtained by solving the equation [52]

$$
\begin{gathered}
\left(I_{m}+1\right) \lambda^{8}+\left(I_{m}-6\right) \lambda^{6}+3\left(I_{m}+2\right) \lambda^{4} \\
-\left(I_{m}+3\right) \lambda^{2}+\lambda^{10}+1=0,
\end{gathered}
$$

see Fig. 2 (red line). The same result can be also obtained directly from our analysis as the condition that the response to the force $\delta f$ is singular [41]; the implied degeneration of the incremental elasticity operator at the boundary [53] is known as the failure of the complementing (ShapiroLopatinskij) condition [54,55].

Note also that a simple lower bound for creasing can be obtained if we compute the value of $\lambda$ beyond which the material becomes physically inextensible. Such limit would follow from the condition $w^{\prime}\left(I_{\lambda}\right) \rightarrow \infty$. For Gent's material we obtain $\lambda_{\text {in }}\left(I_{m}\right)=\sqrt{I_{m}-\sqrt{I_{m}} \sqrt{I_{m}+4}+2} / \sqrt{2}$, and this threshold is also shown in Fig. 2 (black line).

The developed energetic approach to creasing clarifies why this mechanical instability exhibits some features of the second order type phase transition while remaining a fundamentally nonlinear, nucleation type phenomenon. The nonuniversal physical nonlinearities were found to have little influence on the creasing threshold which is largely controlled by the universal geometrical nonlinearity. The proposed methodology should prove useful in other "non-linearizable" problems where local instability is associated with the degeneration of the energy minimum in the strong sense.

We acknowledge the support by the Grants No. ANR-10IDEX-0001-02 PSL (L. T.) and No. AIRC-MFAG-17412 (P. C.). L. T. thanks L. Mahadevan and E. Hohlfeld for helpful discussions.

[1] A. Willis, Int. Cong. Appl. Mech. Lond. 1, 280 (1948).

[2] T. Tanaka, S.-T. Sun, Y. Hirokawa, S. Katayama, J. Kucera, Y. Hirose, and T. Amiya, Nature (London) 325, 796 (1987).

[3] A. Ghatak and A. L. Das, Phys. Rev. Lett. 99, 076101 (2007).

[4] J. Kim, J. Yoon, and R. C. Hayward, Nat. Mater. 9, 159 (2010).

[5] S. Tang, B. Gao, Z. Zhou, Q. Gu, and T. Guo, Soft Matter 13, 619 (2017).

[6] K. Saha, J. Kim, E. Irwin, J. Yoon, F. Momin, V. Trujillo, D. V. Schaffer, K. E. Healy, and R. C. Hayward, Biophys. J. 99, L94 (2010).

[7] Z. Zhou, Y. Li, W. Wong, T. Guo, S. Tang, and J. Luo, Soft Matter 13, 6011 (2017).

[8] B. Dortdivanlioglu and C. Linder, J. Mech. Phys. Solids 125, 38 (2019).

[9] E. P. Chan, J. M. Karp, and R. S. Langer, J. Polym. Sci. B 49, 40 (2011). 
[10] N. Suematsu, K. Sekimoto, and K. Kawasaki, Phys. Rev. A 41, 5751 (1990).

[11] A. Onuki, Phys. Rev. A 39, 5932 (1989).

[12] T. Hwa and M. Kardar, Phys. Rev. Lett. 61, 106 (1988).

[13] E. Hohlfeld and L. Mahadevan, Phys. Rev. Lett. 106, 105702 (2011).

[14] J. W. Hutchinson, Phil. Trans. R. Soc. A 371, 20120422 (2013).

[15] V. Trujillo, J. Kim, and R. C. Hayward, Soft Matter 4, 564 (2008).

[16] J. Dervaux, Y. Couder, M.-A. Guedeau-Boudeville, and M. Ben Amar, Phys. Rev. Lett. 107, 018103 (2011).

[17] A. Gent and I. Cho, Rubber Chem. Technol. 72, 253 (1999).

[18] C. Lestringant, C. Maurini, A. Lazarus, and B. Audoly, Phys. Rev. Lett. 118, 165501 (2017).

[19] B. Budiansky and J. W. Hutchinson, AIAA J. 4, 1505 (1966).

[20] M. Biot, Appl. Sci. Res. 12, 168 (1963).

[21] E. Hohlfeld and L. Mahadevan, Phys. Rev. Lett. 109, 025701 (2012).

[22] W. Hong, X. Zhao, and Z. Suo, Appl. Phys. Lett. 95, 111901 (2009).

[23] Y. Cao and J. W. Hutchinson, Proc. R. Soc. A 468, 94 (2012).

[24] A. D. Ioffe and V. M. Tihomirov, Theory of Extremal Problems (Elsevier, New York, 2009), Vol. 6.

[25] J. M. Ball and J. E. Marsden, Arch. Ration. Mech. Anal. 86, 251 (1984).

[26] J. M. Ball and K. Koumatos, Arch. Ration. Mech. Anal. 219, 89 (2016).

[27] Y. Grabovsky and L. Truskinovsky, J. Nonlinear Sci. 23, 891 (2013).

[28] D. Chen, S. Cai, Z. Suo, and R. C. Hayward, Phys. Rev. Lett. 109, 038001 (2012).

[29] K. B. Broberg, Cracks and Fracture (Elsevier, Cambridge, 1999).

[30] E. Hohlfeld, Phys. Rev. Lett. 111, 185701 (2013).

[31] M. Diab, T. Zhang, R. Zhao, H. Gao, and K.-S. Kim, Proc. R. Soc. A 469, 20120753 (2013).
[32] J. R. Rice, J. Appl. Mech. 35, 379 (1968).

[33] J. Rice and B. Budiansky, Appl. Mech. 40, 201 (1973).

[34] J. Eshelby, J. Elast. 5, 321 (1975).

[35] A. A. Griffith, Phil. Trans. R. Soc. A 221, 163 (1921).

[36] J. Willis, J. Mech. Phys. Solids 15, 151 (1967).

[37] R. W. Ogden, Non-Linear Elastic Deformations (Courier Corporation, 1997).

[38] P. Ciarletta, Nat. Commun. 9, 496 (2018).

[39] M. Singh and A. C. Pipkin, Z. Angew Math. Phys. 16, 706 (1965).

[40] S. Silling, J. Appl. Mech. 58, 70 (1991).

[41] See Supplemental Material at http://link.aps.org/ supplemental/10.1103/PhysRevLett.122.248001 for further details on the theoretical derivation.

[42] A. Flamant, C.R. Hebd. Seances Acad. Sci. 114, 1465 (1892).

[43] J. K. Knowles and E. Sternberg, Arch. Ration. Mech. Anal. 44, 187 (1972).

[44] F. H. Chen and R. T. Shield, Z. Angew Math. Phys. 28, 1 (1977).

[45] P. J. Olver, Arch. Ration. Mech. Anal. 85, 111 (1984).

[46] J. D. Eshelby, Phil. Trans. R. Soc. A 244, 87 (1951).

[47] E. Noether, Nachr. Ges. Wiss. Goettingen Math.-Phys. Kl. 1918, 37 (1918).

[48] J. Li, K. J. Van Vliet, T. Zhu, S. Yip, and S. Suresh, Nature (London) 418, 307 (2002).

[49] L. Jin and Z. Suo, J. Mech. Phys. Solids 74, 68 (2015).

[50] J. Yang, L. Jin, J. W. Hutchinson, and Z. Suo, J. Mech. Phys. Solids 123, 305 (2019).

[51] A. Gent, Rubber Chem. Technol. 69, 59 (1996).

[52] M. Destrade and N. H. Scott, Wave Motion 40, 347 (2004).

[53] N. Muskhelishvili, Singular Integral Equations (Dover, New York, 1992).

[54] M. Agranovich, in Partial Differential Equations IX (Springer, Berlin Heidelberg, 1997), pp. 1-144.

[55] M. Silhavy, The Mechanics and Thermodynamics of Continuous Media (Springer, Berlin Heidelberg, 2013). 